\title{
La Visita General del virrey Toledo en los estudios recientes sobre Charcas
}

\author{
The General Visit of Viceroy Toledo \\ in recent studies on Charcas \\ Daniel O. Quiroga \\ Universidad Nacional Arturo Jauretche/Universidad de Buenos Aires \\ qdani81@gmail.com
}

\section{Resumen:}

El objetivo de este trabajo es realizar un balance del itinerario recorrido por los estudios coloniales sobre el territorio de la Audiencia de Charcas durante las últimas dos décadas en relación con un tema clásico de la historiografía andina: la visita general del virrey don Francisco de Toledo. Para ello, en primer lugar, se procede a revisar las rupturas y continuidades de las nuevas investigaciones en relación con los trabajos de finales del siglo XX. Posteriormente, se remarca la importancia que, cada vez más, ha ido adoptando la perspectiva de la negociación en las interpretaciones dadas a la visita toledana. Finalmente, se sugiere, a partir de la revisión de cómo fue ejecutada la visita al repartimiento de indios de Sipesipe, Cochabamba, que la exploración de la dimensión político-jurídica de la visita general ofrece la posibilidad de formular nuevas preguntas y aproximaciones a un tema clásico de los estudios coloniales.

Palabras clave: visitas de indios, historia de Charcas, Cochabamba, virrey Toledo, reducciones

\begin{abstract}
This article proposes a review of colonial studies of the territory of Audiencia of Charcas, produced over the last two decades and referred to a classic theme of Andean historiography: the general visit of the viceroy Francisco de Toledo. First, a review of changes and continuities between new and late twentieth century research is made, and the increasingly importance of the negotiation perspective of the Toledo visit is highlighted. Finally, from examining how the visit of the repartimiento de indios in Sipesipe (Cochabamba) was carried out, is suggested that including the political-legal dimension in the analysis of the general visit offers the possibility of asking new questions and making new approaches to a classic topic of colonial studies.
\end{abstract}

Keybords: Indian visits, history of Charcas, Cochabamba, Viceroy Toledo, reducciones

Recibido: 30 de mayo de $2020 \cdot$ Aceptado: 11 de agosto de 2020

Autoctonía. Revista de Ciencias Sociales e Historia Vol. IV, N² 2, Julio-Diciembre 2020, 243-256

ISSN 0719-8213 


\section{Introducción}

Para dialogar sobre la trayectoria que han seguido durante las últimas dos décadas los estudios coloniales sobre el territorio de la Audiencia de Charcas, hemos elegido como hilo conductor un tema clásico de la historiografía andina colonial: la visita general al Perú ordenada por el virrey don Francisco de Toledo (1569-1581).

La visita general, inspección ejecutada sobre el terreno entre 1570 y 1575 , ha sido señalada por la historiografía como una pieza clave de la política de gobierno del virrey Toledo; gobierno que, a su vez, se ha interpretado en forma generalizada como el momento de inflexión que marca la consolidación de un orden colonial maduro en los Andes de la segunda mitad del siglo XVI. Ya en los pioneros estudios de la década de 1970, se indicó que la política toledana tuvo como principales consecuencias el relanzamiento de la economía colonial y una profunda reconfiguración de la sociedad indígena. Las primeras investigaciones que trataron la visita general, tanto de manera central como tangencial, tendieron a poner el foco en tres aspectos fuertemente ligados entre sí. En primer lugar, trabajaron el impacto que ocasionó el establecimiento de una nueva tasa del tributo indígena, cuyo aspecto más destacado fue su tendencia a la monetización (Wachtel, 1976; Assadourian, 1979). En segundo lugar, estudiaron la implementación de la mita toledana, el sistema de masiva migración anual forzada hacia Potosí que favoreció la reactivación del centro minero y de la economía virreinal a partir del "subsidio" de la economía campesina indígena (Assadourian, 1979). Y, en tercer lugar, pusieron atención en las reducciones, es decir, la política de reasentamiento y concentración forzada de las poblaciones indígenas en pueblos trazados en base a criterios de urbanismo hispanos (Málaga Medina, 1974). Estos tópicos, abordados tanto de manera particular como entrelazada, despertaron el interés de sucesivas generaciones historiográficas.

A continuación, nos proponemos revisar cuáles han sido los enfoques y orientaciones generales seguidos durante los últimos veinte años en los estudios de la visita toledana a Charcas ${ }^{1}$. Más precisamente, vamos a centrar nuestra atención en las investigaciones que desplegaron sus análisis sobre el territorio que actualmente corresponde al Estado Plurinacional de Bolivia. Luego de identificar continuidades, rupturas y tendencias, reflexionaremos sobre posibles líneas de investigación a seguir en base a una primera exploración de un caso particular: el de la visita general realizada al repartimiento de indios de Sipesipe (actual distrito del departamento de Cochabamba).

\section{Estudios recientes sobre la visita general de Toledo}

Si se dirige la atención a cómo se trabajó la visita general en las investigaciones llevadas adelante durante las últimas dos décadas, se puede observar una continuidad en las orientaciones temáticas (tasa, mita y reducciones siguen siendo los tópicos centrales), pero también ciertas revisiones y reorientaciones en los abordajes adoptados. Repasemos brevemente lo sucedido en torno a las unidades de análisis, las categorías conceptuales 
y, más generalmente, los marcos hermenéuticos utilizados por los estudios coloniales.

Al atender a la escala de análisis, es posible identificar que, salvo escasas excepciones (Mumford, 2017; Zagalsky, 2014), las investigaciones han continuado la tendencia, iniciada a principios de la década de 1980, de alejarse de las visiones de conjunto y privilegiar los enfoques regionales o a micro-escala. De este modo, los trabajos han optado por direccionar su atención hacia unidades como la etnia (Del Río, 2005), la encomienda (Percovich, 2010), el repartimiento de indios (Jurado, 2004; Zagalsky, 2013; Del Río, 2018) o el pueblo de reducción (Palomeque, 2013), y han dejado de lado escalas mayores como Charcas o el virreinato peruano.

Por otro lado, se observa una constante y cada vez más sofisticada crítica de las categorías analíticas empleadas para el estudio del pasado colonial. Buscando una mayor precisión conceptual fueron revisados, discutidos e incluso descartados, conceptos frecuentemente utilizados en la producción historiográfica. Aquí, vale mencionar la fecunda reflexión desplegada en torno a categorías claves como etnicidad, territorialidad (Del Río, 2005), comunidad (Zagalsky, 2009a), tasa y tributo (Percovich, 2010), visitas y revisitas (Zagalsky, 2009b).

Una tendencia similar se vislumbra en las consideraciones metodológicas sobre el uso de las fuentes. El impacto de las teorías vinculadas al análisis del discurso ha signado la hermenéutica de documentos coloniales. De esta manera, recuperando enfoques presentados inicialmente para la región andina durante la década del 1990 (Guevara Gily Salomón, 1994; Tandeter, 1997), las nuevas investigaciones dedican gran atención al contexto de producción de las fuentes y a la intencionalidad de los diferentes sujetos involucrados en su elaboración.

Además de estos tres aspectos, otra cuestión a destacar de los itinerarios historiográficos recientes es el uso generalizado y extendido de la caracterización, promovida ya desde los años 80, de los indígenas como actores sociales. Guiados por la intención de eludir visiones que victimizan de una manera simplificada a los indígenas y avanzar más allá de explicaciones binarias (como las expresadas en las formulaciones dominadores/ dominados y conquista/resistencia), diversas investigaciones destacan el rol activo y creativo de los indígenas frente a las instituciones coloniales. Estos estudios procuran evidenciar y esclarecer las tácticas y estrategias de negociación, adaptación y resistencia desplegadas por los colectivos étnicos frente a las fuerzas de control y sujeción coloniales.

En un esfuerzo por identificar y exponer la capacidad negociadora de los indígenas, los trabajos de los últimos veinte años se fueron preocupando cada vez más por mostrar las ambivalencias, contradicciones y múltiples mediaciones que atravesaron el proceso de visita general. Con algunos matices, esta perspectiva interpretativa se ha empleado para analizar las respuestas dadas a la visita toledana por diferentes colectivos étnicos de Charcas. Así, por citar algunos ejemplos destacables, se sostuvo que los caciques soras establecieron alianzas con las autoridades 
coloniales, ofreciéndoles su lealtad en el cumplimiento de la mita minera a cambio de mantener un control del territorio (Del Río, 2005); se sugirió que los chichas desarrollaron pactos para la visita, tasación y reducción con la autoridades españolas impulsados por el interés compartido en contener el avance de un enemigo común: los chiriguanos (Palomeque, 2013); y se propuso, después de analizar el comportamiento de los visisa en pos de conservar el control de recursos, pensar la negociación como un momento específico dentro de un proceso más extenso de lucha, que podía incluir un variado repertorio de prácticas, como presentaciones judiciales, resistencias larvadas y acomodamientos diversos (Zagalsky, 2013). La perspectiva centrada en la agencia indígena ha sido incorporada, adicionalmente, en trabajos que, corriendo temporalmente su atención a lo acontecido una generación después de la visita de Toledo, sostienen que los colectivos étnicos supieron adoptar y hacer suyas las reducciones y sus instituciones, fundando nuevos pueblos, cabildos y cofradías, y desarrollando efectivas estrategias legales (Penry, 2017).

De igual manera, la visión de un proyecto toledano rígido en lo que concierne a la imposición de la nueva tasa del tributo (Platt et al., 2006: 365-368) ha encontrado algunos matices locales. Novedosos estudios de las tasaciones del tributo a lo largo del siglo XVI, centrados en los valles orientales de La Paz, han revelado notorias continuidades entre la tasa de Toledo y las tasaciones previas que les permiten sugerir la existencia de acuerdos locales sobre la tasación y repensar las visiones historiográficas tradicionales que caracterizaban a la tasa toledana como una normativa inflexible, aplicada de forma uniforme en todo el virreinato peruano (Percovich, 2010).

En suma, las recientes investigaciones historiográficas recurren frecuentemente a conceptos como negociación, pacto, acuerdo, alianza y mediación para dar cuenta de la participación activa de las poblaciones andinas durante el complejo proceso de visita general. Yendo más allá de la dicotomía entre opresión y resistencia, pero sin negar la violencia estructurante del sistema colonial, adoptan, implícita o explícitamente, la premisa de que los visitadores toledanos debieron establecer acuerdos con diversos agentes locales, adaptando o incluso descartando el modelo ideal reflejado en las instrucciones para la visita a fin de cumplir con sus objetivos. Utilizando este enfoque, los estudios coloniales de las últimas décadas consiguieron ampliar la comprensión del complejo proceso de visita toledana en Charcas a la par que abrieron nuevos interrogantes, dirigidos ahora a los términos concretos en que se desarrollaron las negociaciones durante la visita: ¿cuáles fueron las acciones específicamente ejecutadas por los visitadores para ganar la voluntad de los indígenas, más allá de la coerción? ¿Qué cuestiones se podían negociar y cuáles no durante la visita? ¿Qué ofrecían y qué solicitaban las partes involucradas? ¿Cómo afectaba la negociación entre indígenas y visitadores a otros actores locales? Estas y otras preguntas emergen como consecuencia de la creciente relevancia que se le otorga a la negociación indígena.

Los interrogantes mencionados para los estudios dedicados a Charcas se hallan en consonancia con las repercusiones generadas por investigaciones 
referidas a los mismos temas, pero cuyo foco de atención se encuentra en otras regiones del virreinato peruano. Autores de novedosos y sofisticados análisis sobre las reducciones toledanas en el Perú, elaborados desde las disciplinas histórica (Mumford, 2012; Zuloaga Rada, 2012) y arqueológica (Wernke, 2013), debieron especificar lo que entienden por negociación, entre otros motivos, para evitar que sus trabajos sean interpretados como lecturas demasiado celebratorias de la agencia indígena (Saito et al., 2014). Es de suponer que esta demanda por conocer más detalles de los términos en que se desarrollaron las negociaciones entre agentes indígenas y españoles no pueda ser satisfecha totalmente a partir del análisis de las fuentes tradicionalmente empleadas para estudiar la visita general. Las disposiciones dadas por el virrey Toledo, los padrones de indios y los libros de tasa confeccionados, o los resúmenes, son fuentes muy valiosas para identificar los rasgos generales de la visita, pero presentan limitaciones cuando se las quiere utilizar para trabajar los pormenores de la inspección. En consecuencia, se vuelve imprescindible la exploración de otro tipo de fuentes en la búsqueda de nuevos indicios que posibiliten un acercamiento al día a día de la visita general.

Desafortunadamente, las fuentes para abordar este período suelen presentar información fragmentaria, estar dispersas en múltiples repositorios, a veces sin catalogar, o incluso estar ausentes. En este sentido, vale recordar que muchas veces no es posible responder las preguntas que van surgiendo en el campo historiográfico por no disponer de la documentación apropiada. Por ello, es de celebrar cuando una investigación logra dar con una fuente extraordinaria que permite ampliar la comprensión de un tema específico, como sucede con el trabajo recientemente publicado por Luis Glave (2017). En él, se ha conseguido dar mayor detalle de los procedimientos desarrollados por los inspectores generales durante la visita al analizar los libros de cuentas de los visitadores, y ponerlos en relación con otros documentos que contienen información también excepcional, como las cartas que Juan de Matienzo, oidor de la Audiencia de Charcas, envió al rey.

Llamamos la atención sobre este trabajo porque permite advertir un aspecto que no había sido abordado hasta ahora por los estudios dedicados al tema: la potestad de los visitadores generales como administradores de justicia. Al presentar a estos inspectores imponiendo multas, arbitrando en disputas locales y estableciendo sanciones a diferentes actores, Glave recupera la dimensión jurídico-política de la visita general, y exhibe el entrelazamiento existente entre las nociones del gobierno castellano y el ideal de hacer justicia. De cierto modo, la investigación invita a dirigir nuestra mirada al sistema legal colonial para buscar ampliar la comprensión que tenemos de las negociaciones realizadas durante la visita toledana. Tal como sugiere Yannakakis (2013) al revisar la historiografía reciente sobre las prácticas judiciales de los pueblos indígenas de México y los Andes, desde hace tiempo, diversas investigaciones muestran que el sistema legal colonial funcionaba como un facilitador para la negociación entre el gobierno y sus súbditos indígenas. Tomando distancia de los enfoques que entendían al derecho exclusivamente como una herramienta de dominación imperial, los 
más novedosos estudios sobre la participación indígena en la justicia colonial conciben al sistema legal como un escenario de disputa cultural y despliegue del proceso hegemónico, una arena propicia para el encuentro cultural entre formas hispanas e indígenas, la negociación y resolución de conflictos (Yannakakis, 2013; 2015). De ahí que, como observa Cunill (2012), la esfera legal se haya convertido en el espacio preferido por aquellos historiadores que estudian la negociación indígena.

Teniendo en cuenta los antecedentes historiográficos recién mencionados, se puede considerar que un camino fructífero para dar mayores precisiones sobre los pormenores de la visita general y las negociaciones realizadas durante su desarrollo puede ser el estudio de la dimensión jurídico-política de la visita. En lo que sigue, nos interesa realizar una exploración de la potencialidad de esta línea de investigación a partir de la presentación de los primeros avances de un estudio en curso sobre la visita general efectuada a los indios del repartimiento de Sipesipe, en el valle charqueño de Cochabamba.

\section{La visita al repartimiento de Sipesipe}

En 1573, los indios del repartimiento Sipesipe, quienes en las décadas previas habían acumulado una valiosa experiencia como litigantes en variadas disputas por el territorio, aprovecharon la nueva coyuntura abierta por la inspección general para renovar y ampliar sus denuncias por usurpación de terrenos contra españoles. Ante Diego Núñez Bazán, visitador general enviado por el virrey Toledo a los valles de Cochabamba, los indígenas demandaron a más de una docena de personas por ocupar parcelas dispersas en los valles.

Al momento de la visita general, el repartimiento de indios de Sipesipe se encontraba en manos de la Corona Real y estaba disperso en los fértiles e irrigados valles Bajo y Sacaba. La región de Cochabamba tenía la peculiaridad de haber sido el escenario donde el inca Huayna Capac instaló un enclave multiétnico destinado a la elaboración de maíz. Desarticulado el centro productivo por la invasión europea, el vacío social generado fue posteriormente llenado con la aparición de nuevos actores que se enredaron en variados y prolongados enfrentamientos por el acceso a las tierras (Larson, 1992). De las diversas formas que adoptó la lucha por el territorio en esta región, las disputas legales son las que mayores rastros dejaron en los repositorios documentales.

El conocimiento de estos sucesos y sus intentos de resolución en el marco de la visita general es posible gracias a la conservación de diferentes legajos sobre pleitos por tierras en los repositorios de la sección Expedientes Coloniales Cochabamba (ECC) del Archivo Histórico Municipal de Cochabamba (AHMC). La documentación consultada se encuentra distribuida en diferentes volúmenes que contienen información de calidad diversa; en algunos casos, se conservan todas las piezas correspondientes al proceso judicial y, en otros, solamente información fragmentaria. No obstante estas limitaciones y vacíos documentales, una mirada al conjunto del corpus documental permite una aproximación general al desarrollo y resolución de estos conflictos en el marco de la visita toledana. 
Mapa 1. Corregimientos y pueblos de reducción de Charcas en tiempos de Toledo

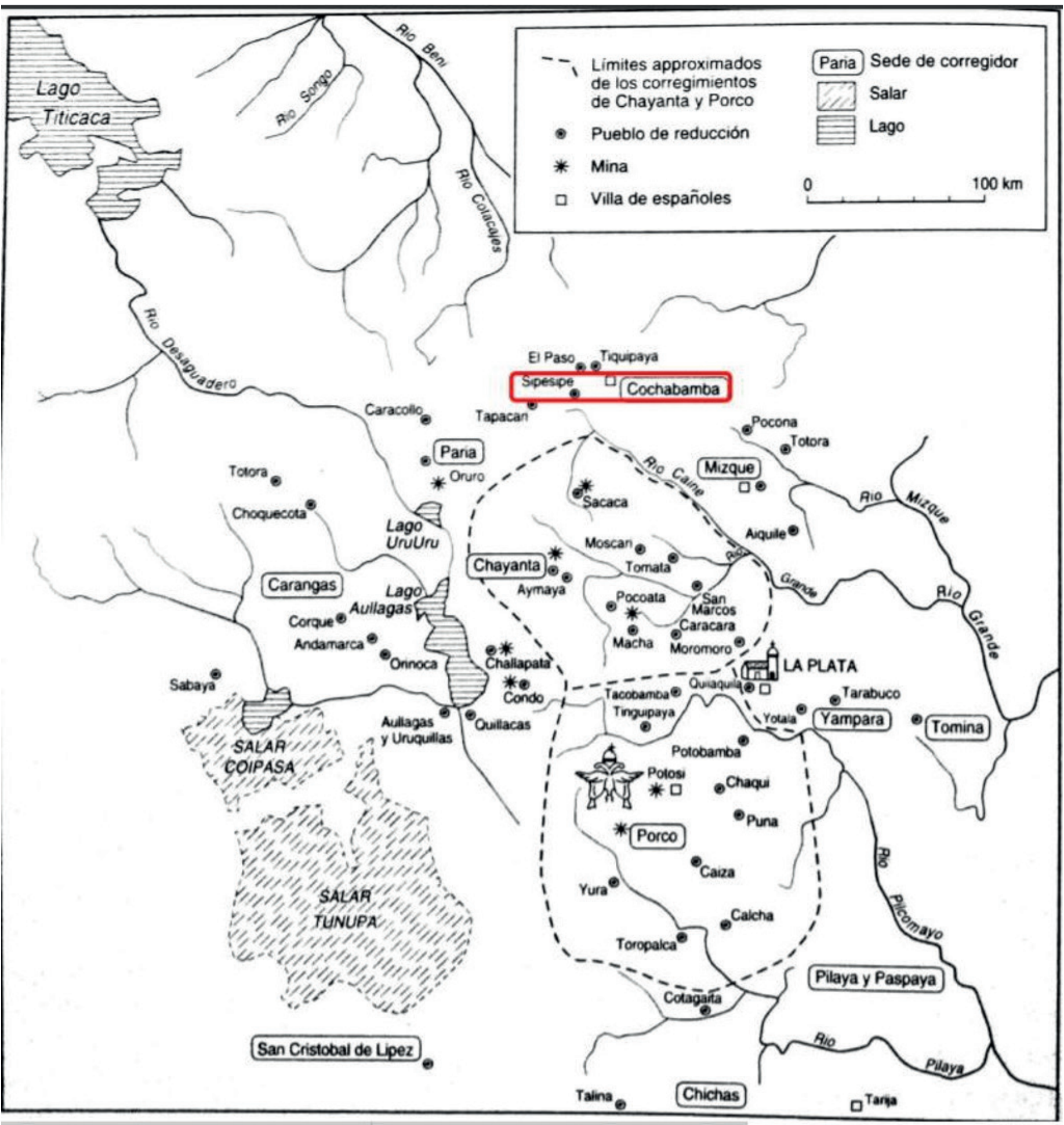

Intervenido sobre Platt et al. (2006: 488) 
Como oficial habilitado para administrar justicia, el visitador Diego Núñez Bazán debió ocuparse de recibir las denuncias de los indígenas, evaluar las probanzasy testimonios presentados por cada una de las partes enfrentadas en los litigios y dictar sentencia. La tarea no fue sencilla. El detalle de las personas y tierras involucradas en los pleitos es extenso ${ }^{2}$. Los indios del repartimiento de Sipesipe demandaron a Garci Ruiz de Orellana, Pedro de Estrada y Francisco Pizarro por tomar tierras en Canata; Diego Mejía de Ovando y Juan Pérez de Ardón por ocupar parcelas en Cala Cala; Juan Sánchez Macías y Fernando de Zárate por usufructuar tierras en Colcapirua; Martín de la Rocha por entrar en los terrenos en Quillacollo; Pedro de Herrera por ocupar las tierras de Tacata; Andrés de Rivera y Diego de Vargas por tomar parcelas en los asientos de Coachaca y Sarico de Viloma; Pedro Vélez de Guevara y los hermanos Gutiérrez de Quirós, Diego y Esteban de Quirós, por ocupar tierras en Coachaca; Juan Flores por entrar en las tierras de Tacona y Francisco Gallegos por tomar las tierras de Chacacollo. Con excepción de estos dos últimos casos, correspondientes a tierras ubicadas en el valle de Sacaba, la mayor parte de los terrenos disputados se encontraban dentro del valle Bajo.

Algunos de los enfrentamientos se arrastraban desde finales de la década de 1550 mientras que otros eran recientes. Una característica común a todos los pleitos fue que hasta ese momento el reclamo de los indígenas no había tenido respuestas favorables. La intervención de Núñez Bazán como administrador de justicia debe haber despertado nuevas expectativas entre los miembros del repartimiento. A continuación se revisará cuál fue el parecer del visitador general hacia finales de 1574 .

Diego Núñez Bazán tomó por válidas las pruebas, presentadas por los indios del repartimiento de Sipesipe, de que eran naturales del valle desde antes de la llegada de los incas, y declaró que el colectivo étnico era el legítimo dueño de las tierras disputadas. No obstante, también reconoció que algunos de los españoles denunciados habían conseguido justos títulos de las tierras litigadas. Tal como se puede observar en la Tabla 1, para poner fin a los conflictos, sin desatender los diferentes tipos de derecho enfrentados, el visitador resolvió que, en la mayoría de los casos, los españoles conservaran y legalizaran la posesión de los terrenos que habían ocupado y usufructuado a cambio del depósito de diferentes sumas de dinero en las cajas de comunidad del repartimiento de indios, en concepto de restitución, y el pago de las costas de la visita general. Únicamente en dos casos ordenó la devolución de las tierras a los indígenas: en el pleito contra Pedro Vélez de Guevara, debido a que el español no poseía justos títulos y el terreno en disputa se encontraba cerca de la reducción de Sipesipe, y en el litigio contra Juan Flores (del cual no tenemos mayores detalles por no haber encontrado el fallo). Finalmente, en otros dos casos, los correspondientes a los pleitos entablados contra Diego de Vargas y los hermanos Gutiérrez de Quirós, Núñez Bazán dictaminó que el primero debía abandonar una parte y, los segundos, todos los terrenos litigados por estar ubicados cerca de la reducción. Pero, en compensación, les otorgó otros que los indígenas habían despoblado en los valles. 
Mapa 2. Valle de Cochabamba

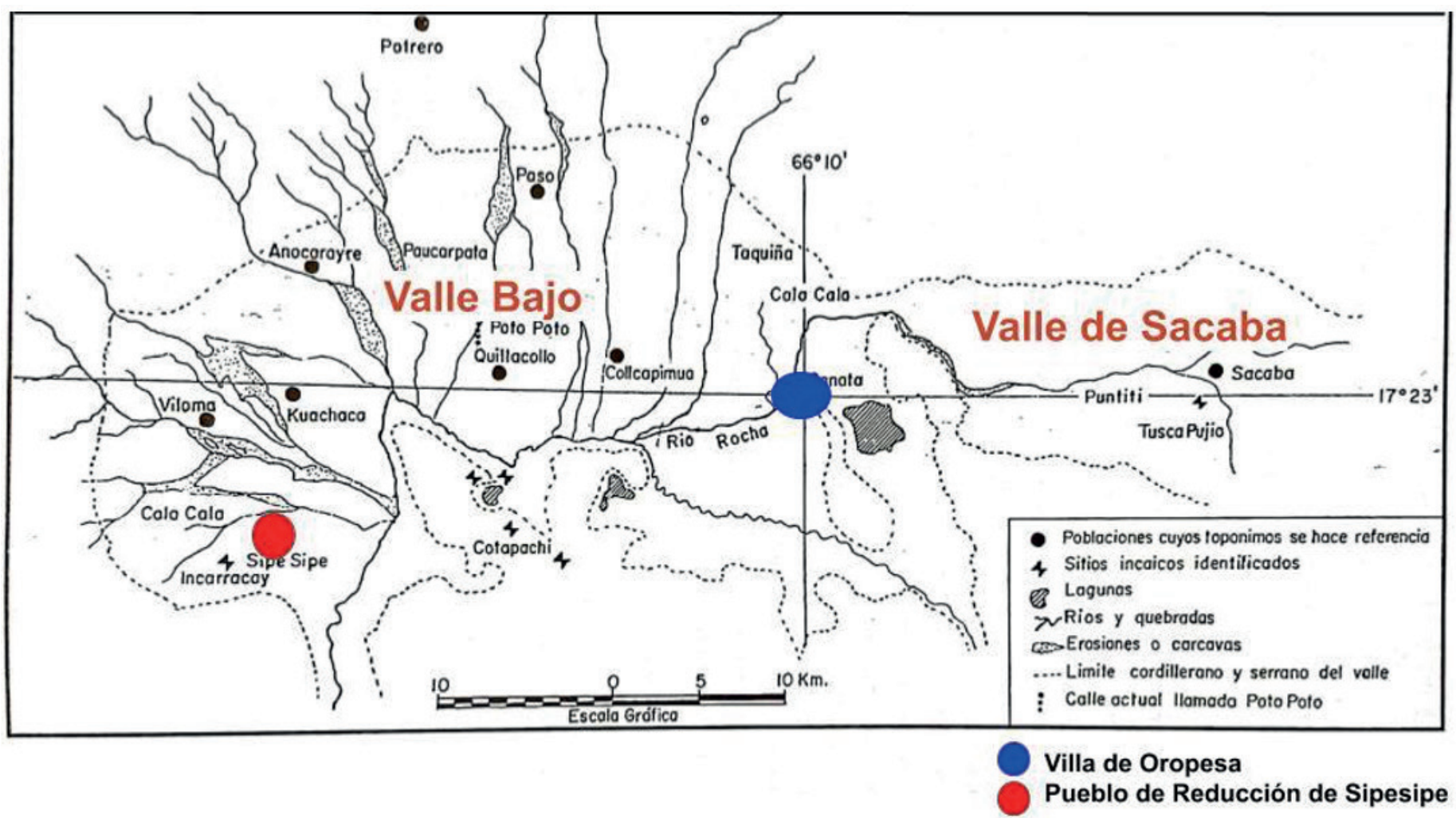

Intervenido sobre Del Río (2005: 56)

Volviendo a las inquietudes originales de profundizar el conocimiento en torno a los pormenores de la visita general y las negociaciones entabladas entre los colectivos étnicos y los visitadores generales: ¿qué tipo de información nos brindan estas fuentes al respecto?, ¿qué indicios de negociación se pueden encontrar en los litigios?, ¿de qué manera el accionar del visitador como juez puede ser leído como parte de una negociación con los actores locales para cumplir con las instrucciones dadas por el virrey Toledo?

A diferencia de otras fuentes frecuentemente utilizadas para estudiar la inspección general, los pleitos por tierras brindan valiosa información de los actores locales -indígenas, españoles y mestizos-, de las disputas entabladas por el acceso a recursos - de su extensión espacial y temporal$\mathrm{y}$, de manera general, de las relaciones de poder existentes al interior de la sociedad colonial.

Los pleitos permiten, además, pensar en los escenarios de la negociación. Así, al revisar los documentos es posible verificar los días y lugares donde el visitador general se estableció para dictar sentencia. Al contrastar el tipo de fallo emitido con el lugar específico donde alternativamente fue firmado - la estancia del español Andrés Rivera en Sarico, la villa de Talavera de Sipesipe (pueblo de reducción de los indios) o el asiento 
Tabla 1. Personas demandadas, tierras en disputa y fallo del visitador general

\begin{tabular}{|l|l|l|}
\hline $\begin{array}{l}\text { Personas demandadas por } \\
\text { los indígenas }\end{array}$ & $\begin{array}{l}\text { Ubicación de tierras } \\
\text { en disputa }\end{array}$ & Fallo del visitador general Núñez Bazán \\
\hline Garci Ruiz de Orellana & Canata & Conserva las tierras a cambio de pagar restitución \\
\hline Francisco Pizarro & Canata & Conserva las tierras a cambio de pagar restitución \\
\hline Juan Pérez de Ardón & Cala Cala & Conserva las tierras a cambio de pagar restitución \\
\hline Juan Sánchez Macías & Colcapirua & Conserva las tierras a cambio de pagar restitución \\
\hline Martín de la Rocha & Quillacollo & Conserva las tierras a cambio de pagar restitución \\
\hline Pedro de Herrera & Tacata & $\begin{array}{l}\text { Conserva las tierras a cambio de entregar ovejas de } \\
\text { castilla en concepto de restitución }\end{array}$ \\
\hline Andrés de Rivera & $\begin{array}{l}\text { Coachaca y Sarico } \\
\text { de Viloma }\end{array}$ & Conserva las tierras a cambio de pagar restitución \\
\hline $\begin{array}{l}\text { Diego de Vargas } \\
\text { de Viloma }\end{array}$ & $\begin{array}{l}\text { Debe ceder parte de las tierras a los indios a cambio de } \\
\text { otras y pagar restitución }\end{array}$ \\
\hline Pedro Vélez de Guevara & Coachaca & Debe abandonar las tierras \\
\hline $\begin{array}{l}\text { Hermanos Gutiérrez de } \\
\text { Quirós, Diego y Esteban } \\
\text { de Quirós }\end{array}$ & Coachaca & $\begin{array}{l}\text { Deben abandonar las tierras a cambio de otras } \\
\text { despobladas por los indios }\end{array}$ \\
\hline Juan Flores & Tacona & Debe abandonar las tierras \\
\hline Francisco Gallegos & Chacacollo & Conserva las tierras sin tener que pagar restitución \\
\hline
\end{tabular}

Elaboración propia a partir de AHMC ECC 4-36, 16-1, 16-6, 16-8, 16-11,17-15,17-22, 19-8, 19-11, 22-18, $24-45,25-41,32-35$ y $45-14$.

de Canata (núcleo de fuerte presencia española en la región) - , se puede corroborar que hubo escenarios más o menos propicios para la negociación indígena. En este sentido, cabe destacar que las sentencias que ordenaban a los españoles abandonar las tierras cercanas al pueblo de reducción fueron firmadas por el visitador cuando este se encontraba presente en el asentamiento indígena de Sipesipe, mientras que los fallos que permitían a los españoles conservar las tierras litigadas fueron firmados en los demás escenarios, de mayor presencia española. De este modo, al pensar la cartografía de las relaciones de poder durante la visita, parece correcto afirmar que el asiento indígena fue el principal escenario donde se desplegó y cristalizó la negociación entre el visitador y los indios del repartimiento.

Ahora bien, para analizar estos pleitos, es preciso tener en cuenta que su desarrollo fue en el marco de la visita general y que Núñez Bazán, al momento de dirimir las disputas, debió contemplar las diversas instrucciones con las que había sido enviado a los valles. En este sentido, conviene recordar que el proyecto toledano para la región de Cochabamba consistía en reconfigurar el territorio - en base a la concentración de los indios en pueblos de reducción y la consolidación de la 
vecindad y población española en torno a la Villa de Oropesa-para desarrollar las potencialidades productivas de la región y transformarla en un “enorme granero" (Assadourian, 1994: 136).

Así, al observar el impacto generado en el territorio por las decisiones tomadas por el visitador general en su rol de juez, es posible afirmar que las sentencias permitieron a los españoles el control de los terrenos situados en los alrededores del recién fundado núcleo hispano y garantizaron al repartimiento el acceso a tierras cercanas al asentamiento indígena que se constituiría, a partir de la visita, en el pueblo de reducción de Talavera de Sipesipe.

La lectura de los pleitos permite incluso sostener que existió una negociación de los indígenas con el visitador para el establecimiento de esta reducción. Esta negociación fue vehiculizada a través de los marcos dados por el sistema de justicia castellano y se plasmó en los litigios por tierras desarrollados durante la inspección general. Como resultado de la negociación, los indios lograron ser reducidos en un asiento que poblaban desde hacía mucho tiempo atrás, a la vez que consiguieron el otorgamiento de derechos a algunas de las tierras que venían reclamando desde hacía varios años y la promesa de entrega de dinero en restitución por aquellas que no les serían devueltas. Quizás esto pueda parecer poco, pero fue mucho más de lo que habían conseguido ante la justicia colonial hasta ese momento. Por su parte, el visitador Núñez Bazán consiguió cumplir con los objetivos del proyecto toledano para los valles cochabambinos de concentrar a los indígenas y liberar otras tierras para el desarrollo productivo destinado a los mercados coloniales.

\section{Comentarios finales}

Los últimos estudios coloniales sobre el territorio de la Audiencia de Charcas han procuraron dar mayor claridad a nuestro conocimiento de la visita general de Toledo. Buscando contestar preguntas formuladas por generaciones historiográficas precedentes, se ha amplificado la dinámica de producción académica. En efecto, la reflexión sobre los indios de Charcas frente a las instituciones coloniales ha dado muestras de gran vitalidad. Adhiriendo al paradigma de la negociación indígena, los trabajos recientes mostraron la complejidad de un proceso que hasta no hace mucho tiempo atrás era visto simplemente como un conjunto de rígidos procedimientos aplicados por las autoridades coloniales.

El abanico de trayectorias para seguir investigando esta problemática continúa ampliándose.

La exploración de la dimensión jurídico-política de la visita general ofrece la posibilidad de formular nuevas preguntas y aproximaciones a un tema clásico de la historiografía sobre Charcas y el virreinato peruano. Consideramos que la búsqueda de nuevas fuentes, relativas al desempeño de los visitadores generales como administradores de justicia, puede abrir el camino a nuevas y fértiles lecturas sobre la visita general del virrey Toledo. 


\section{Fuentes documentales inéditas}

Archivo Histórico Municipal de Cochabamba (AHMC), Expedientes Coloniales Cochabamba (ECC) 4-36, 16-1, 16-6, 16-8, 16-11,17-15,17-22, 19-8, 19-11, 22-18, 24-45, 25-41, 32-35 y 45-14.

\section{Referencias citadas}

Abercrombie, T. (2006): Caminos de la memoria y del poder. Etnografía e historia en una comunidad andina. La Paz, SIERPE.

Assadourian, C. (1979): "La producción de la mercancía dinero y la formación del mercado interno colonial" en Enrique Florescano (comp.), Ensayos sobre el desarrollo económico de México y América Latina, 1500-1975, México, FCE.

Assadourian, C. (1994): "Los derechos a las tierras del Inca y del Sol durante la formación del sistema colonial", en Transiciones hacia el Sistema Colonial Andino, Lima, El Colegio de México-Instituto de Estudios Peruanos, pp. 92-150.

Cunill, C. (2012): “La negociación indígena en el Imperio ibérico: aportes a su discusión metodológica". Colonial Latin American Review, 21:3, pp. 391-412.

Del Río, M. (2005): Etnicidad, Territorialidad y Colonialismo en los Andes: Tradición y cambio entre los soras de los siglos XVI y XVII. La Paz: IEB-IFEA/ASDI-SARIC.

Del Río, M. (2018): “Dinastías señoriales y transformaciones territoriales entre los Sura de Tapacarí S. XVI-XVII", en María de los Ángeles Muñoz (editora), Interpretando huellas. Arqueología, Etnohistoria y
Etnografía de los Andes y sus Tierras Bajas, Cochabamba, INIAM-UMSS.

Gade, D. W. (1991): "Reflexiones sobre el asentamiento andino de la época toledana hasta el presente" en Segundo Moreno Yáñez y Frank Salomon (eds.), Reproducción y transformación de las sociedades andinas, siglos XVI-XX, Quito, Abya-Yala/Movimientos Laicos para América Latina, vol. 1., pp. 69-90.

Gordillo, J. M. y M. Del Río (1993): La visita de Tiquipaya (1573): análisis etno-demográfico de un padrón toledano. Cochabamba, UMSS/CERES/ODEC/FRE.

Guevara Gil, A. y F. Salomon (1994): “A "Personal Visit": Colonial Political Ritual and the Making of Indians in the Andes". Colonial Latin American Review. 3 (1-2), pp. 3-36.

Glave, L. (2017): "La cuadratura del círculo y las rendijas del encierro: política de reducción de indios en los Andes en tiempos del virrey Toledo" en A. Saito y C. Rosas Lauro (editores), Reducciones. La concentración forzada de las poblaciones indígenas en el Virreinato del Perú, Lima, PUCP.

Jurado, C. (2004): "Las reducciones toledanas a pueblos de indios: aproximación a un conflicto", Cahiers des Amériques latines, 47, pp. 123-137.

Larson, B. (1992): Colonialismo y transformación agraria en Bolivia. Cochabamba, 1550-1900. La Paz, CERES/HISBOL.

Málaga Medina, A. (1974): "Las reducciones en el Perú (1532-1600)”. Historia y Cultura, n 8, Lima. 
Mumford, J. (2012): Vertical empire: The Resettlement of Indians in the Colonial Andes. Durham, Duke Universitity Press.

Mumford, J. (2017): “La reducción toledana en el Perú y el Alto Perú, 1569-1575" en A. Saito y C. Rosas Lauro (editores), Reducciones. La concentración forzada de las poblaciones indígenas en el Virreinato del Perú, Lima, PUCP.

Palomeque, S. (2013): “Los chicha y las visitas toledanas. Las tierras de los chichas del Talina (1573-1595)" en A. M. Presta (editora), Aportes multidisciplinarios al estudio de los colectivos étnicos Surandinos. Reflexiones sobre Qaraqara-Charka tres años después, La Paz, Plural/IFEA.

Penry, S.E. (2017): “Pleitos coloniales: 'Historizando' las fuentes sobre pueblos indígenas de los Andes", en A. Saito y C. Rosas Lauro (editores), Reducciones. La concentración forzada de las poblaciones indígenas en el Virreinato del Perú, Lima, PUCP.

Percovich, M. (2010): “Tasa y tributo en la temprana colonia: la encomienda de Songo, Suri y Oyuni en las yungas de La Paz (1545-1573)" en Memoria Americana. Cuadernos de Etnohistoria. 18: 2, Buenos Aires, pp. 149-183.

Platt, T., T. Bouysse-Cassagne y O. Harris (2006): Qaraqara-Charka. Mallku, Inka y Rey en la provincia de Charcas (siglos XV-XVII). Historia antropológica de una confederación aymara. La Paz: IFEA, Plural Editores, University of St. Andrews, University of London, Inter American Foundation, Fundación Cultural del Banco Central de Bolivia.
Rasnake, R. (1989): Autoridad y Poder en los Andes. Los Kuraqkuna de Yura. La Paz, Hisbol.

Saignes, T. (1986): En Busca del Poblamiento Étnico de los Andes Bolivianos (Siglos XV y XVI). La Paz, MUSEF.

Saignes, T. (1991): “Thierry. Lobos y ovejas: formación y desarrollo de los pueblos y comunidades en el sur andino (siglos XVI-XX)”, en Segundo Moreno Yáñez y Frank Salomon (eds.), Reproducción y transformación de las sociedades andinas, siglos XVI-XX, Quito, Abya-Yala/Movimientos Laicos para América Latina, vol. 1., pp. 91-135.

Saito, A., C. Rosales Lauro, J. Mumford, S. Wernke, M. Zuloaga Rada y K. Spalding (2014): "Nuevos avances en el estudio de las reducciones toledanas". Bulletin of the National Museum of Ethnology, 39(1), pp. 123-167.

Tandeter, E. (1997): “Teóricamente ausentes, teóricamente solas. Mujeres y hogares en los Andes coloniales (Sacaca y Acasio en 1614)". Andes. Antropología e Historia, 8, Salta, pp. 11-25.

Wacthel, N. (1976): Los vencidos. Los indios del Perú frente a la conquista española (1530-1570), Madrid, Alianza.

Wernke, S. (2013): Negotiated Settlements: Andean Communities and Landscape under Inka and Spanish Colonialism. Ganesville, University Press of Florida.

Yannakakis, Y. (2013): "Indigenous People and Legal Culture in Spanish America". History Compass, 11/11, pp. 931-947.

Yannakakis, Y. (2015): “Beyond Jurisdictions: Native Agency in the Making of Colonial Legal Cultures". 
A Review Essay. Comparative Studies in Society and History; 57 (4), pp. 1070-1082.

Zagalsky, P. (2009a): “El concepto de 'comunidad' en su dimensión espacial. Una historización de su semántica en el contex to colonial andino (siglos XVI-XVII)". Revista Andina, 48 (Cusco, 2009), pp. 57-90.

Zagalsky, P. (2009b): "Huellas en las revisitas: tensión social e imposiciones coloniales”, en Memoria Americana. Cuadernos de Etnohistoria. 17:2 (Buenos Aires, 2009), pp. 241-279.

Zagalsky, P. (2013): “Tensiones, disputas y negociaciones en torno a la posesión de la tierra. Un mapeo histórico del espacio de los visisa. Andes meridionales, 1570-1610)", en A. M. Presta (editora), Aportes multidisciplinarios al estudio de los colectivos étnicos Surandinos. Reflexiones sobre Qaraqara-Charka tres años después. La Paz, Plural/IFEA.

Zagalsky, P (2014): “La mita potosina: una imposición colonial invariable en un contexto de múltiples transformaciones (Siglos XVI-XVII; Charcas, Virreinato del Perú)". Chungara, Revista de Antropología Chilena. Volumen 46, $\mathrm{N}^{\circ}$ 3, pp. 375-395.

Zuloaga Rada, M. (2012): La conquista negociada: guarangas, autoridades locales e imperio en Huaylas, Perú (1532-1610). Lima, IEP/IFEA.

\section{Notas}

${ }^{1}$ Aunque no corresponden estrictamente al momento historiográfico que nos proponemos revisar, no queremos dejar de mencionar los trabajos de Rasnake (1989), Gade (1991), Saignes (1986, 1991), Gordillo y
Del Río (1993) y Abercrombie (2006) por la importancia que han tenido para ampliar la comprensión de la visita de Toledo y sus repercusiones en las poblaciones indígenas.

${ }^{2}$ Nos basamos a continuación en la información contenida en los siguientes legajos del AHMC ECC: 4-36, $16-1,16-5,16-6,16-8,16-11,17-15,17-22,19-8,19-11$, 22-18, 24-45, 25-41, 32-35 у 45-14.

${ }^{3}$ Cabe aclarar que, si bien hemos conseguido rastrear en el AHMC la mayoría de los fallos emitidos por el visitador general en los litigios por tierras, no logramos encontrar los correspondientes a las demandas entabladas contra Pedro de Estrada, Diego Mejía de Ovando, Fernando de Zárate y Juan Flores. Por ello, en los primeros tres casos no conocemos cuál fue el resultado final de los pleitos. Sin embargo, en el tercero sabemos, gracias a la conservación de una apelación presentada en segunda instancia por Flores contra el dictamen del visitador general, que los indígenas recibieron un fallo favorable. Más allá de estas lagunas documentales, nos parecequela información recopilada permite armar un panorama general de los criterios de justicia aplicados durante la inspección general. 